\title{
Entre la regeneración y la suburbanización: Centro Histórico de Zacatecas, México
}

\author{
Guadalupe Margarita González Hernández \\ Universidad Autónoma de Zacatecas, México \\ Recibido: 15 de junio del 2017 / Aprobado: 5 de septiembre del 2017 \\ doi: 10.26439/limaq2018.n004.2253
}

El Centro Histórico de la ciudad de Zacatecas (CHZ) fue uno de los primeros en forzar su dinámica hacia la regeneración urbana a favor del turismo con el argumento de mejorar las condiciones de vida de sus residentes al tiempo que desplazaba a su población a la periferia. Con el análisis de la evidencia censal (Inegi 1992, 1998, 2000, 2008 y 2010) se llegó a la conclusión de que, a inicios del 2010, el abandono de viviendas, el despoblamiento, la gentrificación, la precarización laboral y el envejecimiento de la población fueron las características más relevantes de una ciudad con vestigios mineros que fue declarada Patrimonio Cultural de la Humanidad en 1993.

Centro Histórico de Zacatecas, suburbanización, gentrificación, segregación, envejecimiento

\section{Between regeneration and suburban development: Historic Center of Zacatecas, Mexico}

The Historic Center of the city of Zacatecas ( $\mathrm{CHZ}$ ) was one of the first to drive its forces towards urban regeneration in favor of tourism with the argument of improving the living conditions of its residents while displacing its population to the periphery. The analysis of census evidence (Inegi, 1992, 1998, 2000, 2008 and 2010) concluded that, at the beginning of 2010, abandonment of dwellings, depopulation, gentrification, job insecurity and population aging were the most relevant characteristics in a city of mining remains declared Cultural Heritage of Humanity in 1993.

Historic Center of Zacatecas, suburban development, gentrification, segregation, aging 


\section{INTRODUCCIÓN}

El presente artículo tiene el objetivo de mostrar cómo la combinación de una política de regeneración urbana con un proceso de suburbanización trajo como consecuencia un centro histórico estancado económica y demográficamente. Este proceso está en concordancia con los objetivos del planeamiento urbano estratégico de activar las zonas en decadencia a través de la gentrificación y el impulso de actividades culturales.

El artículo tiene tres apartados: el primero habla sobre los cambios teóricos y empíricos que han sufrido las ciudades a consecuencia de la aplicación de políticas de regeneración; el segundo refiere a los cambios que ha experimentado el Centro Histórico de Zacatecas (CHZ) en los últimos 30 años; y el tercero establece las conclusiones.

\section{LOS CENTROS HISTÓRICOS COMO ESPACIOS DE INVERSIÓN: TEORÍA Y PRÁCTICA}

\section{Planeamiento racional y estratégico}

En el planeamiento racional, el progreso en manos de la técnica, la ciencia y la tecnología es lo esencial; mientras que las tradiciones, las costumbres, los ritos, las prácticas, las apropiaciones y lo simbólico son representaciones de retraso y conservadurismo. Por ello, el planeamiento urbano se enfocó en que toda necesidad material debía ser satisfecha, y su aplicación implicaba hombres iguales, equivalentes y universales: poseer vivienda, empleo, buena salud, trasladarse fácilmente desde su casa hasta el lugar de empleo y realizar actividades de entretenimiento, como comprar o pasear, era lo básico (Greene, 2005).

Este tipo de planeamiento alude al periodo de la posguerra, en que el Estado benefactor en países europeos o interventor en países latinoamericanos intentó satisfacer las necesidades más generales de la población urbana al proveerle de vivienda, trabajo, transporte y entretenimiento de una forma eficiente y universal en el centro de la ciudad, donde todos convergían. El Estado, por tanto, administraba la ciudad (Harvey, 2006). Entre la escasez 
de recursos y la jerarquización de necesidades, los resultados propiciaron ciudades estructuralmente heterogéneas y desiguales.

La mayoría de los bienes y servicios que satisfacían las necesidades de los habitantes de la ciudad fueron localizados en el centro, lugar adonde convergían desde la periferia consumidores, servicios y bienes. Su jerarquización como centro principal de la ciudad fue apoyada por la densidad de consumidores que lo visitaban, por su poder adquisitivo y por la red de transporte que lo alimentaba (Alegría, 1994).

El cambio de centralidad se efectuó al desplazarse la población hacia la periferia. La dependencia del negocio respecto del cliente (y sus constantes cambios en gustos y preferencias) requirió de su acceso directo a los suburbios (O’Sullivan, 1996). Así se crearon conglomerados de comercios y servicios especializados (malls), donde consumidores redujeron costos de viaje al comparar precios y productos cerca de sus residencias (Goodall, 1977).

Aunque existe variedad en especificidades, se considera que las ciudades estadounidenses son el prototipo, pues ahí se vivió la descentralización de la industria, la reducción de costos en transporte y comunicaciones, los estímulos fiscales, la infraestructura carretera y los precios bajos en la tierra suburbana. La suburbanización de la población siguió al empleo y sus costos de desplazamiento se redujeron. La descentralización de la actividad terciaria fue la fase final (Levy, 1985; O’Sullivan, 1996).

En ciudades con base terciaria, el crecimiento de la población provocó su desplazamiento hacia el perímetro; presionó sus fronteras hacia afuera, a la vez que generó el cambio del uso rural a urbano y su densificación, con secuelas en el mercado inmobiliario. Uno de los factores que indujo a la segregación fue el crecimiento de la población. La invasión de grupos de ingresos medio y bajo en barrios residenciales de alto ingreso en el centro de la ciudad obligó a este grupo social a moverse al borde urbano, con altos costos de transporte, pero con el beneficio de precios bajos de suelo, grandes lotes e infraestructura carretera (Levy, 1985).

El aumento de los gastos públicos en infraestructura urbana y el mejoramiento de la calidad de vida urbana que suscitó la expansión urbana de posguerra 
incitaron a los detractores del planeamiento racional a decir que el Estado debería dedicarse a sus iniciales facultades fiscalizadoras y de seguridad pública. El planeamiento racional dejó de ser útil y surgieron modelos alternativos que estimularon el papel regulador del Estado y favorecieron el surgimiento y la participación de otros agentes sociales en la conducción de los procesos urbanos a inicios de la década de 1980.

Con el planeamiento estratégico, los objetivos fueron inmediatos y fragmentados, y los derechos y las responsabilidades urbanas se distribuyeron entre las esferas pública y privada. El Estado se transformó en un facilitador, un generador de desarrollo local a partir de la coordinación entre las instancias de trabajo y de capital, y el fortalecimiento de los mecanismos participativos en la toma de decisiones. El "nuevo" Estado permitió, en teoría, potenciar los procesos de empoderamiento y de gobernanza local (Greene, 2005); y el planeamiento urbano requirió políticas de coalición y alianzas de clase que organizasen el espacio bajo un complejo sistema de fuerzas movilizadas por diversos agentes sociales (Harvey, 2006).

La expansión urbana se convirtió en fragmentación urbana: zonas y comunidades locales; centro y suburbios; región metropolitana y municipalidades. Y la gobernanza consistió en descifrar el poder real de la reorganización de la vida urbana, más allá de los Gobiernos locales y la administración, que apenas cumplen un papel facilitador y coordinador (Harvey, 2006). En ese sentido, el poder de organizar el espacio urbano se origina de un complejo conjunto de fuerzas movilizadas por los diversos agentes sociales, en que el conflicto y la coalición se entrelazan en aras de revitalizar ciertas áreas (Poria y Ashworth, 2009).

\section{Rehabilitación física, revitalización económica y conducta social}

El proceso expansivo urbano causó estancamiento y declinación en las áreas centrales, problema que se resolvió con tres estrategias. Estas se describen a continuación.

La rehabilitación fisica rescata las estructuras fisicas de la zona. De acuerdo con planes de manejo, el Estado planifica qué edificios rehabilitar para mejorar la calidad de vida. El capital inmobiliario invierte en edificios en mal estado y 
los convierte en centros comerciales, oficinas o viviendas de mejor calidad. Los grupos sociales que viven en dichas zonas aparentemente son reubicados de manera temporal mientras rehabilitan esos lugares. No obstante, ante el aumento del precio del uso del suelo que provoca la rehabilitación, los pobladores optan por salirse definitivamente (Linares y Lan, 2007).

Estos proyectos son discutidos por la sociedad, bajo la bandera de la gobernanza urbana (referéndums, consultas, participaciones), pero son auspiciados por agentes económicos y políticos interesados en impulsar la zona (McCarthy, 2003). Los posibles conflictos se desvanecen al tratar la problemática de forma individual o al manejar pequeños grupos con intereses diferenciados.

El impacto se da en el mejoramiento económico y la segregación. Con viviendas mejoradas, la renta del suelo se incrementa y solo puede ser pagada por grupos con mayor ingreso y bagaje cultural. Como consecuencia, se pierden la mezcla social y la identidad de la zona para adquirir otras nuevas, bajo la dinámica del estilo de vida del grupo dominante en esta nueva fase (Bourdieu, 2003).

La revitalización económica crea actividades como el turismo y el comercio, o servicios que interesan a visitantes y residentes. Desde esta lógica, las ciudades entran en competencia para atraer capital y ofrecer ventajas físicas, económicas y sociales. Su imagen es el mecanismo para vender y los índices de competitividad y calidad de vida son instrumentos comparativos con el resto del mundo (Snepenger et al., 2003).

El Estado, al igual que en la rehabilitación fisica, cumple un papel esencial en esta estrategia: crea infraestructura, marcos legales e incluso inversión inicial para impulsar la revitalización económica. El capital privado, después de las pérdidas iniciales, es el continuador para mantener su estado competitivo.

Los tradicionales distritos comerciales son espacios de interacción entre residentes y visitantes; pero las interacciones e impactos del turismo pueden tener efectos nocivos: los residentes empiezan a entender que los comercios y los servicios satisfacen las necesidades de los turistas. Las actividades culturales, de entretenimiento y ocio llegan a ser artificiales, y los espacios públicos se rehabilitan para los visitantes. El distrito tradicional llega a ser evitado por residentes por carecer de significado real, lo cual también afectará con el paso del tiempo a los turistas (Snepenger et al., 2003). 
El punto central de la estrategia híbrida es la cultura y la creatividad como fábricas de cohesión social en la zona central, apoyadas por la rehabilitación fisica y la revitalización económica. El concepto de ciudad creativa viene de una movilización de la inherente creatividad en arte y cultura para establecer nuevas industrias y oportunidades de empleo, al mismo tiempo que suscita una lucha contra los problemas ambientales, raciales, étnicos y de los sin hogar (Sasaki, 2010).

Según esta estrategia, el actual desarrollo del capitalismo se dirige a una nueva fase distintiva, en la cual las fuerzas impulsoras de la economía no son solo tecnológicas u organizacionales, sino humanas. La clase creativa (un concepto bastante vago, que comprende trabajos intensivos en el conocimiento y cuya función es crear nuevas formas significativas: artistas, científicos, analistas, gerentes de negocios, creadores de opinión) es una "clase dominante en la sociedad". Las personas de esta clase son distinguidas por sus recompensas materiales (altos salarios) y desean vivir con calidad en excitantes lugares, ser siempre creativos y tolerantes. Los planificadores involucrados en reactivar zonas centrales priorizan ambientes creativos e imágenes de ciudades cool para atraer a dichos profesionales (Zimmerman, 2008).

Además del arte y la cultura, se desarrolla la rehabilitación fisica y la revitalización económica con enormes implicaciones políticas y sociales. Los sectores inmobiliario y gubernamental construyen caracterizaciones simbólicas en publicidad y mercadotecnia para legitimar sus acciones, atraer inversiones, empleadores, residentes y visitantes. Crean representaciones del espacio público bajo nuevos paisajes urbanos, como la construcción de infraestructura de transportes y comunicación, parques temáticos e industriales, centros de convenciones, centros comerciales, estadios deportivos y salas de conciertos (Kim, 2010).

El valor de estas construcciones fisicas y simbólicas se convierte en capital cultural solo si cumple los lineamientos de dicha clase creativa. Bajo ese precepto, los sitios históricos, patrimoniales y creativos pueden ser mercancías en tanto la cultura y el arte se convierten en productos sustentables para la sociedad y la economía urbana (Wang, 2009). Mas la discriminación y la exclusión para quienes no congenian con dichos intereses está garantizada (Sasaki, 2010). 
A pesar de las especificidades, los resultados son similares: la atracción de residentes, capitales y visitantes refiere a un mejoramiento alusivo al estrato social y a la competitividad económica de estas zonas bajo las características de volatilidad, superficialidad, individualidad y diversidad, en detrimento de la universalidad y la inclusión social.

Con esta práctica, una nueva estructura espacial desigual emergió, y la disparidad entre la ciudad central, la inherente y los suburbios llegó a ser mayor. Las áreas rehabilitadas provocaron un aumento del precio del suelo, en tanto, las zonas periféricas y los suburbios ofrecieron la tasa de gradiente del suelo más baja (Wang y Yu-Lee, 2009). Casi siempre, los proyectos de rehabilitación fueron otorgados a grandes empresas desarrolladoras, las que promovieron un estilo arquitectónico que facilitó la atracción de grupos sociales de alto ingreso. Muchas de las áreas centrales se transformaron gradualmente en bienes residenciales para élites. El redesarrollo urbano junto con el desplazamiento de la pobreza son presentados como una estrategia de hacer el lugar y, así, un mecanismo para incrementar los valores del suelo.

La acción municipal, por tanto, no necesita ajustar la estructura de impuestos, perseguir a las grandes corporaciones para tentarlas a invertir y proveer infraestructura urbana básica; solo suministra el tipo adecuado de infraestructura material y cultural (Zimmerman, 2008), que la sumerge en deudas impagables (Harvey, 2006).

La relevancia del grupo creativo y sus prácticas culturales, como objeto de atracción urbana al final expresan la invisibilidad del resto de los estratos sociales, en especial el de la clase trabajadora (Zimmerman, 2008). Esta política de regeneración urbana impulsó los grupos diversos (feministas, gays, solteros) y ocultó-desplazó los grupos desiguales (desempleados, trabajadores, jornaleros, estructuras familiares tradicionales).

La disminución de funciones tradicionales (administración pública, comercio y servicios) y el impulso de la economía creativa son resultado de la mercantilización de la cultura asociada al entretenimiento y ocio. Las zonas rehabilitadas y revitalizadas se convierten en lugares donde los bares, antros, restaurantes y centros de espectáculos dominan y segregan a grupos sociales ajenos (Roberts, 2006). 


\section{CASO DE ESTUDIO: SUBURBANIZACIÓN, GENTRIFICACIÓN Y ENVEJECIMIENTO EN EL CENTRO HISTÓRICO DE ZACATECAS}

La ciudad de Zacatecas-Guadalupe (CZG) se localiza aproximadamente 600 kilómetros al norte de la Ciudad de México. Su origen se remonta al periodo colonial, época en la que fue uno de los centros mineros más relevantes. El Centro Histórico de la ciudad de Zacatecas (CHZ) está localizado al noroeste de la zona urbana y hasta el 2003 fue su principal centro económico, social, político y cultural, y con mayor carga simbólica. Aunque la mayor parte de su diseño arquitectónico y urbanístico proviene de mediados del siglo XIX -excepto la catedral o el Palacio de Gobierno, construidos en el siglo XVIII-, es considerado un lugar que expresa el poderío minero novohispano.

Si bien la regeneración urbana se aplicó desde 1980, cabe precisar que la última etapa ha sido significativa por proveer una partida exclusiva del presupuesto estatal para el fomento de la actividad turística (Gobierno del Estado de Zacatecas, 2007-2012). La partida presupuestal supera por mucho cualquier inversión desarrollada en el CHZ desde que se consideró polo de impulso económico y cultural, y representa en promedio, el $19 \%$ del gasto programable del presupuesto estatal anual (Gobierno del Estado de Zacatecas, 2010).

Aunque tiene tres partidas etiquetadas (creación y modernización de infraestructura turística; fortalecimiento de la difusión y promoción turística; y creación y mejoramiento de los servicios turísticos), cabe esperar cualquier cosa en términos de dirigir los recursos. No existe un rigor en criterios de planificación y ejecución, mucho menos transparencia en la rendición de cuentas en pro del turismo. El destino del recurso público para promoción y generación de espectáculos con el fin de atraer turistas se ha centrado en la promoción, pero bajo el velo está el despilfarro.

Aunque los recursos presupuestarios tienen el objetivo de convertir el estado de Zacatecas en destino turístico, la mayor parte se destinó exclusivamente al fomento del CHZ (Gobierno del Estado de Zacatecas, 2012). En el 2008, el $78 \%$ de dicho rubro presupuestal se centró en el CHZ; en el 2009 se incrementó a $81 \%$; en el 2010 a 85 \%; en el 2011 a 88 \%, y en el 2012 a 90 \% (Gobierno del Estado de Zacatecas, 2008-2012). 
Su objetivo es exclusivamente el fomento turístico, lo que lleva a la exclusión de proyectos de fomento económico de otro tipo, como los de comercio o de servicios no turísticos. Como se demuestra líneas abajo, las actividades comerciales y de servicios mostraron visos de estancamiento y tendencia a desplazarse hacia la periferia en busca de mercados cautivos que el CHZ ya no ofrecía.

A pesar de las fuertes inyecciones de inversión pública, el turismo como sector económico no despuntó en el periodo 1990-2010. La creación de establecimientos, los empleos, los ingresos derivados de la actividad, el valor agregado y las remuneraciones al trabajador estuvieron totalmente estancados.

Finalmente, el nombramiento de Patrimonio Cultural de la Humanidad por norma impidió cambios en la estructura del CHZ; situación que no fue obstáculo para que el capital inmobiliario desde finales de la década de los noventa haya dejado fachadas intactas y modificado interiores con el fin de cambiar su uso para actividades turísticas más rentables, lo cual propició discrepancias con instancias estatales controladoras del patrimonio.

La Unesco no tuvo disponibilidad o autoridad para compartir la conservación urbana de Zacatecas. Aunque el apoyo de la Unesco fue valorado, prestigiado y, en muchos aspectos, fijó la agenda para la conservación del patrimonio, la realidad fue diferente. El poder de la Unesco para conservar el patrimonio urbano fue condicionado y diluido por autoridades y empresarios locales bajo la tutela de la Junta de Protección y Conservación de Monumentos y Zonas Típicas del Estado de Zacatecas (JPYCMYZTEZ). El Estado usó el poder político y económico para llevar a cabo la conservación urbana, bajo el control de los empresarios locales.

En el CHZ los funcionarios locales hicieron todo lo posible para hacer el ambiente compatible con la preocupación de la Unesco por la autenticidad. Después del nombramiento, sin embargo, esta influencia quedó afuera por dos razones: primero, los consultantes culturales afiliados a la Unesco no pudieron intervenir ampliamente en las relaciones domésticas zacatecanas; y segundo, no produjeron adecuados recursos económicos para forzar a los Gobiernos estatal y municipal a seguir sus consejos. 
Durante dos décadas, entre 1990 y 2010, el CHZ presentó las siguientes características:

1. Despoblamiento y envejecimiento. El despoblamiento fue producto, en un primer momento, de la suburbanización, principalmente al norte de la ciudad de Guadalupe (desde Bernárdez hasta La Condesa), donde familias jóvenes encontraban viviendas de interés social a un precio más bajo que el de las viviendas localizadas en la parte central de la ciudad de Zacatecas. Este proceso estuvo acompañado por una descentralización de la actividad económica desde el CHZ hasta la zona Bernárdez, localizada en el sector limítrofe entre los municipios de Zacatecas y Guadalupe.

En un segundo momento fue la caída de la tasa (de por sí baja) de crecimiento geométrica desde inicios de la década de 1990 en la CZG: 0,865 para el periodo $1990-1995$; 0,631 para 1995-2000; 0,68 para 20002005; y en el 2005-2010, de 0,68. Esto fue producto de la emigración hacia otras áreas urbanas y la propensión de las familias jóvenes a tener menos hijos, lo anterior aunado a la caída de la tasa de mortalidad incrementó la esperanza de vida a tal grado que la tendencia es al envejecimiento de su población residente (González, 2010).

A diferencia de lo vivido en otras áreas de la CZG, el CHZ mostró desde 1990 una caída de residentes (36 \%), así como del dinamismo demográfico. El fenómeno de despoblamiento del $\mathrm{CHZ}$ se originó en aquellos grupos que refieren a edades infantil (disminuyó $48 \%$ ), juvenil $(57 \%)$ y adulto-joven (34 \%). Ante ello, el grupo de adultos mayores tuvo mayor relevancia: en 1990 solo era el 4 \% de la población del CHZ, y en el 2010 concentró el $12 \%$.

Pese a ello, por su magnitud, la composición demográfica estuvo dominada por el grupo de edad adulta (18-59 años) como resultado de que la CZG vivió su bono demográfico durante el periodo 1990-2010. Este fenómeno contrasta con el proceso vivido en las áreas periféricas de la mancha urbana, donde los grupos de edades infantil y juvenil fueron dominantes. Durante este lapso, el CHZ se convirtió en un espacio urbano solitario, donde la mayoría de sus residentes tendían a envejecer. 
2. Aumento de la población con mayor instrucción educativa. La población de la CZG mejoró su grado de instrucción educativa: en 1990, un zacatecano estudiaba 9,4 años en promedio, en el 2000 esta cifra subió a 9,675 y en el 2010 a 10,4. El CHZ fue el área con los valores más altos: en 1990, 11,2 años estudiados; en el 2000, 11,4 años; en el 2005, 12,06 años; y en el 2010, 12,35 años. Tales valores están clasificados en educación terciaria o superior.

En 1990, el $31 \%$ de la población mayor de 18 años y más que vivía en el CHZ adquirió educación superior y esta cifra se incrementó a $44 \%$ en el 2010. Al mejorar su nivel educativo, los residentes del CHZ presentaron demandas económicas, sociales, políticas y culturales más sofisticadas.

3. Segregación socioespacial por ingreso. El proceso de segregación socioespacial-gentrificación en el CHZ se caracterizó por la expulsión (ya sea por políticas de rehabilitación física o por los altos precios de rentas del suelo) de pobladores con ingresos bajos y en el fomento de viviendas para pobladores de ingresos altos, de preferencia con estructuras familiares pequeñas y con gustos más sofisticados por la cultura y las artes. Este proceso, llamado por muchos gentrificación (concepto castellanizado del inglés por el que se identificó un cambio de las estructuras sociorresidenciales en las zonas centrales de las ciudades y la variación de un grupo de ingreso bajo y étnico-racial afroamericano por un grupo de ingreso alto y étnico-racial caucásico), está relacionado con un proceso de aislamiento socioespacial de este grupo en detrimento de la mezcla social, sin considerar la variable étnica, a causa de ser irrelevante en esta zona urbana, que mostró un proceso de mestizaje mayor que el $98 \%$ de la población total en el periodo de análisis (1990-2010).

La mezcla social de los distintos grupos sociales o racial-étnicos es esencial para el buen funcionamiento de las áreas urbanas. El dominio de uno en términos económicos, culturales, políticos o sociales en el espacio urbano alude a un proceso de segregación del resto de los grupos o autosegregación en tanto desea autoexcluirse del resto.

Desde 1990, el CHZ se vio afectado en su mezcla social, producto del ingreso económico. Por medio del índice de segregación socioespacial (Alegría, 1994b, 423), se observó, desde 1995 hasta el 2010, el dominio del 
grupo social con ingresos mayores de dos y hasta cinco salarios mínimos (SM), al mostrar un índice cercano a 0 (mayor mezcla social) con un valor de 5. Hasta 1995, el grupo de ingreso dominante fue el que recibió menos de dos SM al presentar un índice de 12.

Espacialmente, a pesar de su despoblamiento, el CHZ y su área vecinal no sufrieron una importante pérdida de población ocupada que recibe por actividad laboral más de cinco salarios mínimos. El CHZ y su área vecinal fueron durante el periodo de 1990-2010 lugares atractivos para que residan personas pertenecientes al grupo con ingresos mayores de cinco salarios mínimos; mucho más atractivos que para el grupo con ingresos de dos y hasta cinco salarios mínimos. El CHZ y su área vecinal concentraron el mayor número de personas residentes clasificadas en este grupo de ingreso alto (más de cinco salarios mínimos) durante dicho periodo (25\% en 1990 y $45 \%$ en el 2010).

Para resumir, el CHZ y su área vecinal mostraron en 20 años condiciones suficientes para afirmar que hubo un proceso de segregación, el cual se basó en dos fenómenos: a) segregación de los grupos de ingresos bajos (menor de dos salarios mínimos) a los barrios de menor equipamiento urbano y localización geográfica (Los Bolos y Pánfilo Natera), donde se concentraron los valores más bajos de instrucción educativa, población ocupada y con derechohabiencia a seguridad social y viviendas habitadas; b) autosegregación de los grupos de ingresos medios (de dos y hasta cinco salarios mínimos) y altos (más de cinco salarios mínimos). Con comportamientos similares en indicadores de hacinamiento, empleo, seguridad social y nivel de instrucción educativa, aunque con decisiones de localización residencial distintas (el grupo de ingreso medio se concentró dominantemente en los barrios de Alameda, El Tanquecito y La Ciudadela, mientras que el de ingreso alto se acomodó en la zona central y en los barrios de Rebote y Sierra de Álica), ambos grupos mostraron su poder cultural, económico y físico en el área de análisis.

Desde esa lógica, la segregación socioespacial está presente en el área con dos dinámicas: a) dominio demográfico, social y económico de personas que reciben ingresos mayores de dos y hasta cinco SM, lo cual impone comportamientos culturales, sociales y políticos, así como demanda de 
bienes y servicios; y b) Su dominio espacial al concentrar la mayor parte de las propiedades. Este grupo dominante decidió localizarse en la parte oeste del CHZ. A lo largo del periodo 1990-2010, dicho grupo social evitó la mezcla social con los grupos sociales que no obtuvieron ingresos o recibieron ingresos menores de dos SM.

4. Situación de la vivienda. En relación con las condiciones físicas de la vivienda (calidad de paredes, techos y pisos; dotación de servicios, como agua, drenaje, energía eléctrica), hacinamiento y equipamiento urbano, el CHZ fue el área mejor equipada de la CZG durante el periodo 19902010. Sin embargo, el fenómeno de mayor relevancia fue la vivienda deshabitada.

Las viviendas deshabitadas están relacionadas con el mercado inmobiliario mexicano, que favoreció la vivienda nueva para trabajadores con ingresos mayores de dos SM y con derechohabiencia a la seguridad social del Instituto Mexicano del Seguro Social (IMSS) o del Instituto de Servicios de Seguridad Social de los Trabajadores del Estado (ISSSTE) (González, 2013).

A esto se añadió la suburbanización de la población y las políticas de conservación del CHZ. La suburbanización de la población en la CZG estuvo supeditada al comienzo del uso del suelo agrícola y minero para la construcción de viviendas e infraestructura vial y urbana a mediados de 1970. La construcción de viviendas de interés social y residencial permitió la expansión hacia la ciudad de Guadalupe, soportada por la construcción de avenidas e instalación de sistemas de servicios públicos básicos (alumbrado público, agua potable, drenaje) a escala mayor.

Del CHZ se empezaron a desplazar a los residentes y se atrajo la actividad económica. Fue el centro principal económico de la CZG hasta el 2003. A partir del 2008, se evidenció el traslado de la centralidad económica hacia un lugar más acorde con las nuevas dinámicas de la economía regional, nacional e internacional: zona Bernárdez.

Durante el apogeo económico del CHZ (de 1980 al 2003), la renta del suelo se disparó. Los precios pagados por las actividades económicas eran superiores hasta en $800 \%$ a los pagados en otras áreas de la ciudad. Al aumentar la renta del suelo, para el 2005 la actividad económica, excepto 
el turismo, abandonó el CHZ para seguir a los consumidores periféricos. En 1988, el CHZ contribuyó con el 18,8 \% del PIB de la CZG; en el 2008 solo contribuyó con el 0,06 \% e inauguró un periodo de abandono y estancamiento en la conservación de la vida residencial.

La suburbanización dejó mansiones coloniales deshabitadas. Algunas de ellas, localizadas en el primer cuadro del $\mathrm{CHZ}$, fueron adaptadas para la administración pública, el comercio, los servicios y el turismo. Las que no cumplieron los requisitos de localización estratégica fueron abandonadas.

La CZG posee un marco legal de protección al patrimonio natural y colonial desde 1965 (Ley de Protección y Conservación de Monumentos y Zonas Típicas del Estado de Zacatecas). Bajo la tutela de un grupo de "interesados" en el cuidado del patrimonio cultural (influyentes empresarios inmobiliarios), se creó la JPYCMYZTEZ. Esta entidad, junto con el Instituto Nacional de Antropología e Historia (INAH) y la Organización de las Naciones Unidas para la Educación, la Ciencia y la Cultura (Unesco), se encargaron de proteger y mantener la estructura del CHZ, lo cual devino en su nombramiento de Patrimonio Cultural de la Humanidad en 1993.

Con estos lineamientos inflexibles, los dueños de las casas, las casonas y las mansiones no tuvieron muchas opciones para adecuarlas a los nuevos patrones de comportamiento basados en la movilidad y nuevas tecnologías de comunicación y transporte. La mayoría de las edificaciones simplemente fueron abandonadas por el alto costo de mantenimiento y la baja demanda de uso. El CHZ para el 2010 se convirtió en el área con mayor número de viviendas deshabitadas de la mancha urbana (18 000).

Como la tenencia del suelo es privada, las decisiones de utilización y rehabilitación de los edificios lo son de igual forma. En 1990, en el CHZ el $90 \%$ de las viviendas eran particulares; para 1995 se incrementaron a $91 \%$; en el 2000 a $96 \%$; en el 2005 a 99 \%; y en el 2010 a 99,8 \%. Por tanto, si bien las políticas de conservación y mantenimiento en el CHZ fueron discutidas, planeadas y ejecutadas en el ámbito público, la influencia de los propietarios de las edificaciones históricas fue contundente, sobre todo de aquellos que concentraban más propiedades. 
A esto hay que sumar la caída de los índices de ocupación. El promedio de personas por vivienda en el CHZ descendió de 4,6 en 1990 a 3,3 en el 2010, contra los 4,3 y 3,5, respectivamente de la CZG; valores que sustentan aún más su despoblamiento.

5. Empleo y seguridad social. Por la centralidad económica del CHZ, el empleo fue esencial en su dinámica interna. Ahí se demandaron trabajadores del resto de la mancha urbana para sufragar su actividad. En el 2003, producto del cambio de la centralidad económica, la demanda de trabajadores empezó a retraerse y desplazarse hacia el nuevo centro, Bernárdez, por lo que se dio un cambio radical en la composición social y del empleo en el CHZ.

El primer cambio se evidenció en la disminución de la población económicamente activa (PEA), producto del despoblamiento y el aumento de la población adulta mayor que caracteriza el lugar; en 20 años cayó en 350 \%. La población ocupada (PO) también descendió, aunque a ritmos menos acelerados. Finalmente, la población desempleada aumentó debido a lo siguiente: deterioro de las condiciones socioeconómicas en el CHZ; estancamiento económico de la ciudad y del país en el periodo 19902010. La población desempleada representó el $1 \%$ en 1990; mientras que en el 2010, el $10 \%$ de la PEA.

Con respecto a la seguridad social, por la caída de la $\mathrm{PO}$, el número de residentes del $\mathrm{CHZ}$ con derecho a seguridad social disminuyó también. Durante un periodo de 20 años, sus residentes perdieron el derecho a la seguridad social debido al aumento del desempleo (la derechohabiencia está ligada al empleo) y la precarización laboral (acceso a trabajo con ingresos magros y sin prestaciones sociales). De 1990 al 2010, la proporción de PO del CHZ descendió $32 \%$, en tanto la proporción de población con derecho a seguridad social cayó $69 \%$.

Adicionalmente, el CHZ, al igual que la CZG, deterioraron la calidad de la seguridad social, debido a la disminución de la población cubierta por el ISSSTE combinada con un incremento de población cubierta por IMSS y Seguro Popular, y con el aumento de población sin derechohabiencia. Para 1990, el ISSSTE, junto con seguridad social al 61 \% de su población; 
en el 2010 solo lo hizo con el 25 \%. La nueva dinámica laboral privilegió la seguridad social de los trabajadores privados cubiertos por el IMSS, donde por definición presentaban mayor precariedad laboral y social que aquellos asegurados por el ISSSTE.

El aumento de la población cubierta por el IMSS en el CHZ a partir de 1995 indica la diversificación de la ocupación económica de la PEA en sectores privados y sociales más que en el sector público. Al inicio del periodo 1990-2010, la mayor parte de la PO se orientaba a la administración pública. Ante el adelgazamiento del Estado, al final de dicho periodo se produjeron recortes de personal (y por tanto de seguridad social) que era absorbido principalmente por el sector social (Seguro Popular) y privado (IMSS); la menor parte de la PO fue deteriorando su condición al grado de abultar el desempleo y la proporción de personas sin derecho a la seguridad social.

Además, el patrón de segregación en la localización residencial se intensificó. En la parte noroeste del CHZ se incrementaron los residentes con seguridad social cubierta por IMSS; en tanto, en el sur y el este, a pesar de su disminución, permaneció el mayor conglomerado de personas que cubrían su seguridad social con el ISSSTE.

6. Especialización económica en el CHZ. Al inicio del periodo 19901993, el CHZ se concentró en ofrecer todos los productos que requería la CZG, pero era mucho más productivo en el comercio al por menor y en los servicios básicos que requerían principalmente los pobladores (además de los servicios turísticos). Para 1995-1998, se redujeron las ramas económicas ofertadas en el CHZ, a causa de que la actividad turística irrumpió con bonanza. De hecho, el comercio perdió capacidad, aunque siguió siendo relevante. Durante estos años, se desplazaron a su área vecina la mayoría de los bienes y servicios básicos ofrecidos en el CHZ.

Entre el 2000-2003 la actividad turística perdió competitividad ante el comercio al por menor y sobre todo ante los servicios de entretenimiento, industria filmica, televisiva y radiofónica, así como los culturales y financieros. Hasta este periodo, el CHZ concentraba mayor capacidad para crear economías de escala, de alcance y aglomeración que el resto 
de la mancha urbana. Es decir, los precios y los productos ofrecidos en el CHZ eran los más competitivos de la CZG; situación que hizo posible aumentar la escala en el tamaño de los negocios establecidos en su área. Así también, se propiciaron las condiciones para que ahí funcionaran negocios complementarios y sustitutos de los bienes y servicios que se habían ofrecido en el periodo 1995-1998.

En el periodo 2005-2008 se modificó esta situación: las ramas económicas que abastecían bienes y servicios básicos (como servicios al hogar, personales, profesionales, legales o educativos) perdieron su competitividad y productividad en el CHZ y su área vecinal, por lo que se desplazaron hacia la periferia. En este sector estaba su mercado, la población de ingresos bajos, que ya se había instalado en la periferia de la mancha urbana. Asimismo, ramas que requerían de gran accesibilidad y consumidores de altos ingresos (servicios de telecomunicaciones, tecnologías móviles, industria televisiva, filmica o radiofónica) se localizaron en un lugar más cercano a su población objetivo, la zona Bernárdez.

\begin{tabular}{|c|c|c|c|}
\hline 1990-1993 & 1995-1998 & $2000-2003$ & 2005-2008 \\
\hline $\begin{array}{l}\text { Comercio al por } \\
\text { menor de todo } \\
\text { tipo de productos, } \\
\text { servicios turísticos, } \\
\text { de mantenimiento } \\
\text { a los hogares, } \\
\text { profesionales, } \\
\text { médicos, } \\
\text { educativos y de } \\
\text { alquiler }\end{array}$ & $\begin{array}{l}\text { Comercio al } \\
\text { por menor de } \\
\text { todo tipo de } \\
\text { productos, servicios } \\
\text { inmobiliarios, } \\
\text { médicos, } \\
\text { personales, } \\
\text { turísticos, } \\
\text { profesionales y } \\
\text { educativos }\end{array}$ & $\begin{array}{l}\text { Comercio al por } \\
\text { menor de todo tipo de } \\
\text { productos, servicios de } \\
\text { telecomunicaciones, } \\
\text { de entretenimiento, } \\
\text { culturales y de transmisión } \\
\text { radiofónica, televisiva } \\
\text { y fílmica, de internet, } \\
\text { financieros, legales, } \\
\text { inmobiliarios, de empleo, } \\
\text { educativos, médicosy } \\
\text { de cuidado de la salud, y } \\
\text { turísticos }\end{array}$ & $\begin{array}{l}\text { Comercio al por menor de } \\
\text { artículos de lujo asociados } \\
\text { a la salud, medioambiente } \\
\text { y trabajo artesanal; } \\
\text { servicios médicos y de } \\
\text { cuidado de la salud, } \\
\text { turísticos, de alimentación } \\
\text { gourmet; entretenimiento, } \\
\text { de juego y culturales; y } \\
\text { asociaciones culturales, } \\
\text { políticas y sociales }\end{array}$ \\
\hline
\end{tabular}

Tabla 1. Ramas económicas más importantes en el CHZ (1990-2008)

Fuente: González, 2009; G. González; J. González, 2012 
El CHZ solo concentró el $8 \%$ de la provisión y los negocios se especializaron en actividades que requerían de contacto cara a cara, en servicios culturales, de ocio y entretenimiento, solicitados por los pobladores de ingresos altos. En especial, surgieron los restaurantes gourmet, las galerías de arte y los comercios de productos artesanales, lo cual implicaba un cambio en los patrones de consumo, auspiciado por el aumento de residentes y visitantes de ingresos altos.

La tabla 1 ha dado cuenta del cambio de patrón de consumo de los visitantes y residentes del CHZ, y de su área vecinal. A inicios de la década de 1990, el CHZ aún disponía de demanda y oferta de bienes y servicios de todo tipo, que indicaban un centro donde convergían distintos estratos o grupos sociales con patrones de consumo y poderes adquisitivos distintos. A finales de la primera década del siglo Xxi, la especialización del CHZ en venta, distribución y producción de artículos de lujo como comida gourmet, en servicios asociados a lo artesanal y la actividad cultural expuso a un residente con un alto poder adquisitivo, gustos más sofisticados y demandas culturales más complejas. La composición especializada en la oferta económica del CHZ estaba más asociada con el residente que con el turista, pues de acuerdo con una investigación precedente (González, 2014) este último no tuvo un gran impacto en su economía y diversificación. El residente con un mayor nivel de instrucción (educación superior), edad mediana (de 30 a 60 años) y con ingresos mayores de cinco salarios mínimos fue el que cambió la orientación económica del CHZ hacia el fomento cultural y artesanal.

\section{CONCLUSIONES}

El CHZ mostró en 20 años dos fenómenos:

- Segregación de los grupos de ingresos bajos (menores de dos salarios mínimos) al área de menor equipamiento urbano y con lejana localización geográfica, donde se concentraron los valores más bajos de instrucción educativa, población ocupada, derechohabiencia a seguridad social y viviendas habitadas. 
- Autosegregación de grupos de ingreso medio (de dos y hasta cinco salarios mínimos) y alto (más de cinco salarios mínimos) con comportamientos similares en hacinamiento, empleo, seguridad social y nivel de instrucción educativa, aunque con decisiones de localización residencial distintas: el primer grupo se concentró en la parte oeste, y el de ingreso alto se acomodó en el sur y el este. Lo más importante fue que ambos grupos mostraron su poder cultural, económico y físico en el área de análisis.

En el CHZ, al igual que en otros centros históricos, se ha consolidado la gentrificación con rasgos específicos:

- Posicionamiento discreto pero contundente del grupo de ingreso alto, en contrapartida con la expulsión de los grupos de ingresos bajos hacia la periferia, lo cual ha cambiado los patrones de consumo y la oferta económica del lugar.

- Mejoramiento de los niveles educativos al pasar del dominio de la primaria terminada en 1990 al de la educación superior en el 2010.

- Disminución importante del promedio de personas por vivienda. Esto provoca que las casonas sean habitadas por tres personas, quienes disponen de grandes espacios para diversas actividades $\left(200 \mathrm{~m}^{2}\right.$ en promedio). Esta situación contrasta con la periferia, donde en viviendas de $45 \mathrm{~m}^{2}$ en promedio viven de tres a cuatro personas.

- Un aún dominante grupo de personas de entre 18 y 59 años, demandantes de bienes y servicios específicos que aluden a la tendencia del $\mathrm{CHZ}$ a especializarse en actividades culturales y de entretenimiento. Al mismo tiempo, un grupo cada vez más importante de personas de 60 años a más predomina en el espacio del CHZ.

- Despoblamiento, producto de varios factores combinados (suburbanización de la población joven, con familia y de bajos ingresos; política inflexible de cambiar la estructura física del CHZ; descentralización de la actividad económica e impulso de la actividad turística) que intensifica el deterioro fisico, cultural y económico del área. 
Hasta la fecha no existe un proyecto de repoblamiento inducido. Las políticas de rehabilitación física y desarrollo económico (en especial, de entretenimiento y ocio) en los últimos 20 años han propiciado condiciones idóneas para impulsar el turismo y gentrificar el CHZ. Esto ha dado como consecuencia un ambiente adecuado para la exclusión de grupos sociales distintos de los que perciben ingresos medios y altos, fenómeno que se compagina con un estancamiento económico, suscitado por la caída en la demanda de bienes y servicios cotidianos, así como por el privilegio de contar con servicios de entretenimiento y el ocio.

\section{REFERENCIAS}

Alegría, T. (1994). Condiciones espaciales de la pobreza urbana y una propuesta para su disminución. Frontera Norte [número especial], 1(6), 61-76.

Alegría, T. (1994b). Segregación socioespacial urbana. El ejemplo de Tijuana. Estudios Demográficos y Urbanos, 9(2), 411-428.

Bourdieu, P. (2003). La distinción. Criterios y bases sociales del gusto. México:Taurus.

Gobierno del Estado de Zacatecas. (2008-2012). Cuentas públicas 20082012. Zacatecas: Gobierno del Estado de Zacatecas.

González, G. (2009). Centralidad y distribución en el ingreso. Cambios en la estructura de la ciudad Zacatecas-Guadalupe (1990-2004). Zacatecas: Conacyt / Universidad Autónoma de Zacatecas.

González, G. (2013). La paradoja del derecho a la vivienda en México: vivienda urbana nueva deshabitada, 1990-2010. Semina: Ciências Sociais e Humanas, 34(1).

González G. (2014). Circo sin pan. Regeneración y mercantilización en el Centro Histórico. Ciudad de México: Universidad Nacional de Zacatecas. 
González, G., y González, J. (2012). Capital y trabajo: implicaciones de la reestructuración productiva en el espacio. Delos: Desarrollo Local Sostenible, 5(15).

Goodall, B. (1977). La economía de las zonas urbanas. Madrid: Instituto de Estudios de Administración Local.

Greene, R. (2005). Pensar, dibujar, matar la ciudad: orden, planificación y competitividad en el urbanismo moderno. Eure, 31(94), 77-95.

Harvey, D. (2006). A produção capitalista do espaço. São Paulo: Annablume.

Instituto Nacional de Estadística y Geografia. (1992). XI Censo General de Población y Vivienda 1990. Zacatecas.

Instituto Nacional de Estadística y Geografia. (1996). Conteo 95. Resultados definitivos. Tabulados básicos. Aguas Calientes: Instituto Nacional de Estadística y Geografia.

Instituto Nacional de Estadística y Geografía. (2000). XII Censo General de Población y Vivienda 2000. Sistema para la Consulta de Información Censal (Scince) 2000.

Instituto Nacional de Estadística y Geografia. (2008). II Conteo de Población y Vivienda 2005 Zacatecas.

Instituto Nacional de Estadística y Geografia. (2010). XIII Censo General de Población y Vivienda 2010.

Kim, Ch. (2010). Place Promotion and Symbolic Characterization of New Songdo City, South Korea. Cities, 27(1).

Levy, J. (1985). Urban and Metropolitan Economics. Nueva York: Mc Graw-Hill.

Linares, S., y Lan, D. (2007). Análisis multidimensional de la segregación socioespacial en Tandil (Argentina) aplicando SIG. Investigaciones Geográficas, (44).

O’Sullivan, A. (1996). Urban Economics. Bostón: Irvin/McGraw Hill. 
Poria, Y., y Ashworth, G. (2009). Heritage Tourism-Current Resources for Conflict. Annals of Tourism Research 36(3).

Roberts, M. (2006). From "Creative city" to "No-go Areas". The Expansion of the Night-Time Economy in British Town and City. Cities (23).

Sasaki, M. (2010). Urban Regeneration Through Cultural Creativity and Social Inclusion: Rethinking Creative City Theory Through a Japanese Case Study. Cities (27).

Snepenger, D., Murphy, L., O'Connell, R., y Gregg, E. (2003). Tourist and Residents Use of a Shopping Space. Annals of Tourism Research 30(3).

Wang, J. (2009). “Art in capital”: Shaping Distinctiveness in a Culture-Led Urban Regeneration Project in Red Town, Shanghai. Cities (26).

Wang,J., yYu-Lee, S. (2009). Gentrification and Shanghai’s New Middle-Class. Another Reflection in the Cultural Consumption Thesis. Cities (26).

Zimmerman, J. (2008). From Brew Town to Cool Town: Neoliberalism and the Creative City Development Strategy in Milwaukee. Cities (25). 